\title{
A CHARACTERIZATION OF STRONGLY MEASURABLE PETTIS INTEGRABLE FUNCTIONS
}

\author{
J. J. UHL, JR. ${ }^{1}$
}

\begin{abstract}
Theorem. Let $\mathfrak{X}$ be a Banach space and $(\Omega, \Sigma, \mu)$ be a finite measure space. A strongly measurable $f: \Omega \rightarrow \mathfrak{X}$ is Pettis integrable if and only if there exists a Young's function $\Phi$ with $\lim _{t \rightarrow \infty} \Phi(t) / t=\infty$ such that $x^{*} f \in L^{\Phi}(\mu)$ for all $x^{*} \in \mathfrak{X}^{*}$.
\end{abstract}

In 1969, Brooks [1] gave a characterization of strongly measurable Pettis integrable functions by use of the Orlicz-Pettis theorem. The purpose of this note is to give another characterization in terms of Orlicz spaces. Throughout this note $\mathfrak{X}$ is a Banach space with continuous dual $\mathfrak{X}$ *. $(\Omega, \Sigma, \mu)$ is a fixed finite measure space. $f: \Omega \rightarrow \mathfrak{X}$ is called strongly measurable if it is the $\mu$-almost everywhere limit of simple functions of the form $\sum_{i=1}^{n} x_{i} \chi_{E_{i}}$ where $x_{i} \in \mathfrak{X}, E_{i} \in \Sigma$ and $\chi_{E_{i}}$ is the characteristic or indicator function of $E_{i}$. A strongly measurable $f: \Omega \rightarrow \mathfrak{X}$ is called Pettis ir.tegrable if $x^{*} f \in L^{1}(\mu)$ for all $x^{*} \in \mathfrak{X}^{*}$ and for each $E \in \Sigma$ there is $x_{E} \in \mathfrak{X}$ satisfying $x^{*}\left(x_{E}\right)=\int_{E} x^{*} f d \mu$ for all $x^{*} \in \mathfrak{X}^{*}$. A Young's function is an increasing convex function $\Phi$ defined on $[0, \infty)$ such that $\Phi(0)=0$. The Orlicz space $L^{\Phi}(\mu)$ is the space of all (equivalence classes of) functions $f$ such that $\int_{\Omega} \Phi(|f| / k) d \mu<\infty$ for some $k>0$.

The following theorem gives the promised characterization of Pettis integrable strongly measurable functions. In a sense this theorem is an analogue of LaVallée Poussin's theorem for vector valued functions.

TheOREm. Let $f: \Omega \rightarrow \mathfrak{X}$ be strongly measurable. $f$ is Pettis integrable with respect to $\mu$ if and only if there exists a Young's function $\Phi$ such that

(i) $\lim _{t \rightarrow \infty} \Phi(t) / t=+\infty$, and

(ii) $x^{*} f \in L^{\Phi}(\mu)$ for each $x^{*} \in \mathfrak{X}$.

Proof (Sufficiency). Suppose $x^{*} f \in L^{\Phi}(\mu)$ for all $x^{*} \in \mathfrak{X}^{*}$. Then if $T: \mathfrak{X}^{*} \rightarrow L^{\Phi}$ is defined by $T\left(x^{*}\right)=x^{*} f, T$ is linear. Furthermore $T$ is closed since if $x_{n}^{*} \rightarrow x^{*}$ in $\mathfrak{X}^{*}$ and $T\left(x_{n}^{*}\right) \rightarrow g$ in $L^{\Phi}(\mu)$ norm, then $x^{*} f=g$ a.e. $[\mu]$ because $x_{n}^{*} f \rightarrow x^{*} f=g$ pointwise almost everywhere. According to Banach's closed graph theorem, $T$ is continuous. Hence if $g \in L^{\Psi}$ where $\Psi$ is

Received by the editors July 6, 1971.

AMS 1969 subject classifications. Primary 2850, 3035.

${ }^{1}$ Supported in part by National Science Foundation grant GP-28577.

(c) American Mathematical Society 1972 
complementary to $\Phi$, and $S(g)$ is defined on $\mathfrak{X}^{*}$ by

$$
S(g)\left(x^{*}\right)=\int_{\Omega} g x^{*} f d \mu, \quad x^{*} \in \mathfrak{X}^{*},
$$

then $S$ is linear in $g$. Moreover for $x^{*} \in \mathfrak{X}^{*}$,

$$
\left|S(g)\left(x^{*}\right)\right|=\left|\int_{\Omega} g x^{*} f d \mu\right| \leqq\|g\|_{\Psi}\left\|x^{*} f\right\|_{\Phi} \leqq\|g\|_{\Psi}\left\|x^{*}\right\|\|T\| .
$$

Hence $S(g) \in \mathfrak{X}^{* *}$ and $S(g)$ is the "Gelfand" integral of $g f$.

Next we shall show that $S$ actually maps bounded functions in $L^{\Psi}$ into the closed subspace $\mathfrak{X}$ of $\mathfrak{X}^{* *}$. To this end, set $E_{n}=\{\omega:\|f(\omega)\| \leqq n\}$. If $g$ is supported in one of the $E_{n}$ 's, say $E_{n_{0}}$, consider

$$
S(g)\left(x^{*}\right)=\int_{\Omega} g x^{*} f d \mu, \quad x^{*} \in \mathfrak{X}^{*} .
$$

Since $f$ is bounded on $E_{n_{0}}, g f$ is actually Bochner integrable. Hence

$$
S(g)\left(x^{*}\right)=x^{*}\left(\text { Bochner }-\int_{\Omega} g f d \mu\right)
$$

i.e. $S(g)=$ Bochner $-\int_{\Omega} g f d \mu$ for $g$ supported inside one of the $E_{n}$ 's. Now condition (i) implies $\Psi(t)<\infty$ when $t<\infty$. Hence by [4, Theorem 10.3, p. 87], $\lim _{\mu(E) \rightarrow 0}\left\|g \chi_{E}\right\|_{\Psi}=0$ for all $g$ in the closed subspace of $L^{\Psi}$ determined by the simple functions. Since $E_{n} \uparrow \Omega$, the continuity of $S$ implies $S(g)=$ $\lim _{n} S\left(g \chi_{E_{n}}\right)$ strongly in $\mathfrak{X}^{* *}$ for all $g$ in the closed subspace of $L^{\Psi}$ determined by the simple functions. In particular, $S\left(\chi_{E}\right)=\lim _{n} S\left(\chi_{E \cap E_{n}}\right)=$ $\lim _{n}$ Bochner $-\int_{\Omega} \chi_{E} \chi_{E_{n}} f d \mu \in \mathfrak{X}$. Hence $S\left(\chi_{E}\right)\left(x^{*}\right)=\int_{\Omega} \chi_{E} x^{*} f d \mu$ and $f$ is Pettis integrable.

(Necessity). Suppose $f$ is Pettis integrable. By a theorem of Pettis [6, Corollary 2.51, p. 284], $\lim _{\mu(E) \rightarrow 0} \int_{E}\left|x^{*} f\right| d \mu=0$ uniformly for $x^{*}$ in the unit ball of $\mathfrak{X}^{*}$. Also $\sup _{\left\|x^{*}\right\| \leqq 1}\left\|x^{*} f\right\|_{L^{1}}<\infty$. An appeal to LaVallée Poussin's theorem [5, Theorem 22, p. 19] establishes the existence of a convex function $\Phi$ defined on $[0, \infty)$ which is nonnegative, increasing, convex and satisfying $\lim _{t \rightarrow \infty} \Phi(t) / t=+\infty$ such that

$$
\sup _{\left\|x^{*}\right\| \leqq 1} \int_{\Omega} \Phi\left(\left|x^{*} f\right|\right) d \mu<\infty .
$$

By setting $\Phi_{1}(t)=\Phi(t)-\Phi(0)$, one finds that $\Phi_{1}$ is a Young's function satisfying (i) and $x^{*} f \in L^{\Phi_{1}}(\mu)$ for all $x^{*} \in \mathfrak{X}^{*}$. This completes the proof of the theorem. 


\section{REFERENCES}

1. James K. Brooks, Representations of weak and strong integrals in Banach spaces, Proc. Nat. Acad. Sci. U.S.A. 63 (1969), 266-270. MR 43 \#266.

2. N. Dunford and J. T. Schwartz, Linear operators. I: General theory, Pure and Appl. Math., vol. 7, Interscience, New York, 1958. MR 22 \#8302.

3. E. Hille and R. S. Phillips, Functional analysis and semi-groups, rev. ed., Amer. Math. Soc. Colloq. Publ., vol. 31, Amer. Math. Soc., Providence, R.I., 1957. MR 19, 664.

4. M. A. Krasnosel'skiǐ and Ja. B. Rutickiř, Convex functions and Orlicz spaces, GITTL, Moscow, 1958; English transl., Noordhoff, Groningen, 1961. MR 21 \#5144; MR 23 \#A4016.

5. Paul A. Meyer, Probability and potentials, Blaisdell, Waltham, Mass., 1966. MR 34 \#5119.

6. B. J. Pettis, On integration in vector spaces, Trans. Amer. Math. Soc. 44 (1938), 227-304.

7. A. C. Zaanen, Linear analysis, measure and integral, Banach and Hilbert space, linear integral equations, Interscience, New York; North-Holland, Amsterdam; Noordhoff, Groningen, 1953. MR 15, 878.

Department of Mathematics, University of Illinois, Urbana, Illinois 61801 